\title{
Overview on Clinical Relevance of Intra-Tumor Heterogeneity
}

\author{
Giorgio Stanta* and Serena Bonin \\ DSM, Department of Medical Sciences, University of Trieste, Trieste, Italy
}

Today, clinical evaluation of tumor heterogeneity is an emergent issue to improve clinical oncology. In particular, intra-tumor heterogeneity (ITH) is closely related to cancer progression, resistance to therapy, and recurrences. It is interconnected with complex molecular mechanisms including spatial and temporal phenomena, which are often peculiar for every single patient. This review tries to describe all the types of ITH including morphohistological ITH, and at the molecular level clonal ITH derived from genomic instability and nonclonal ITH derived from microenvironment interaction. It is important to consider the different types of ITH as a whole for any patient to investigate on cancer progression, prognosis, and treatment opportunities. From a practical point of view, analytical methods that are widely accessible today, or will be in the near future, are

OPEN ACCESS

Edited by:

Stefano La Rosa,

Centre Hospitalier Universitaire Vaudois (CHUV), Switzerland

Reviewed by: Kenji Notohara, Kurashiki Central Hospital, Japan

Maria Grazia Tibiletti, Ospedale di Circolo e Fondazione Macchi, Italy

*Correspondence: Giorgio Stanta stanta@impactsnetwork.eu

Specialty section:

This article was submitted to Pathology,

a section of the journal

Frontiers in Medicine

Received: 16 January 2018

Accepted: 19 March 2018

Published: 06 April 2018

Citation:

Stanta G and Bonin S (2018) Overview on Clinical Relevance of Intra-Tumor Heterogeneity. Front. Med. 5:85.

doi: 10.3389/fmed.2018.00085 evaluated to investigate the complex pattern of ITH in a reproducible way for a clinical application.

Keywords: intra-tumor heterogeneity, morphohistological intra-tumor heterogeneity, clonal intra-tumor heterogeneity, functional phenotypic plasticity, stochastic plasticity, cancer spreading, genomic instability

\section{BACKGROUND}

Today, the knowledge and the clinical evaluation of tumor heterogeneity are extremely important to improve clinical oncology. Inter-tumor heterogeneity exceeds the boundaries of specific tumors and also of their molecular classifications $(1,2)$, which makes the clinical approach very complex. However, the most complex issue is intra-tumor heterogeneity (ITH) as a spatial and temporal phenomenon more or less distinct in every single patient. This is closely related with cancer progression, resistance to therapy, and recurrences. Because of ITH in primary tumors and metastases, and because of the wide clinical heterogeneity among patients, it is necessary to apply clinical research methods directly to patients' material in today's clinical practice to be able to better define a specific effective treatment. For any type of tumor, only few molecular biomarkers are being used in diagnostics at the moment, and a minor part of available treatment targets is applied. Hopefully, wider clinical research directly performed on patients will be increasingly diffused as a requirement in the near future, with the goal to obtain more efficient and personalized therapy protocols (3). Moreover, phase three clinical trials in oncology have recently encountered wide criticisms (4-6), because of the long time, the high cost, and not always satisfying results. They are essentially based on a patient's randomization process, which is unable to cover the entire range of clinical heterogeneity. Sophisticated methods of analysis allow penetration in the very high complexity of cancer biology; in clinical research, those methods can be directly applied on the patient to cover variability in toto. Therefore, only a wide clinical application of research methods can lead to a correct interpretation of the clinical reality. New types of clinical research approaches that consider heterogeneity have recently been suggested in oncology. Those approaches, such as N1 trials, basket, umbrella, and platform studies, have been proposed to 
overcome the limits of classical clinical trials and to shorten the time for a wide clinical application $(7,8)$.

In most papers, when it is reported about ITH, it is mostly dealt with clonal genetic evolution only, but ITH itself is a very complex matter because it is related to different sources and shows different patterns. Recently, also heterogeneity of drug distribution has been shown to be relevant in cancer treatment (9). There are several types of ITHs that can be observed on the morphological-histological and molecular level (Figure 1). All these should be taken into consideration when research is applied to clinics, because each patient needs to be considered as a whole also from this point of view for more effective and reproducible analyses.

On the morphological level, different histological heterogeneity patterns with different levels of differentiation are frequently observed in the same tumor, and it is well known that apart areas of the same tumor can have different patterns of gene expression also without any clonal evolution, e.g., the central part of the tumor compared to the external border (10). If these aspects of ITH are ignored, this can affect the reproducibility of clinical analyses and misinterpretation of the results.

On the molecular level, it is possible to distinguish at least two large categories of ITH, one of which is mostly clonal, transmitted to the daughter cells, and the other one is functional nonclonal (11-13). Recent literature reports have especially focused on the genetic clonal evolution of tumors based on DNA mutations and copy number alterations (CNAs). There is a lower extent of information on epigenetic evolution, which is also mostly clonal. Gene promoter methylation, general hypomethylation of tumor DNA, and histone methylation and deacetylation are very common in cancer and are as relevant as genetic alterations (14). The

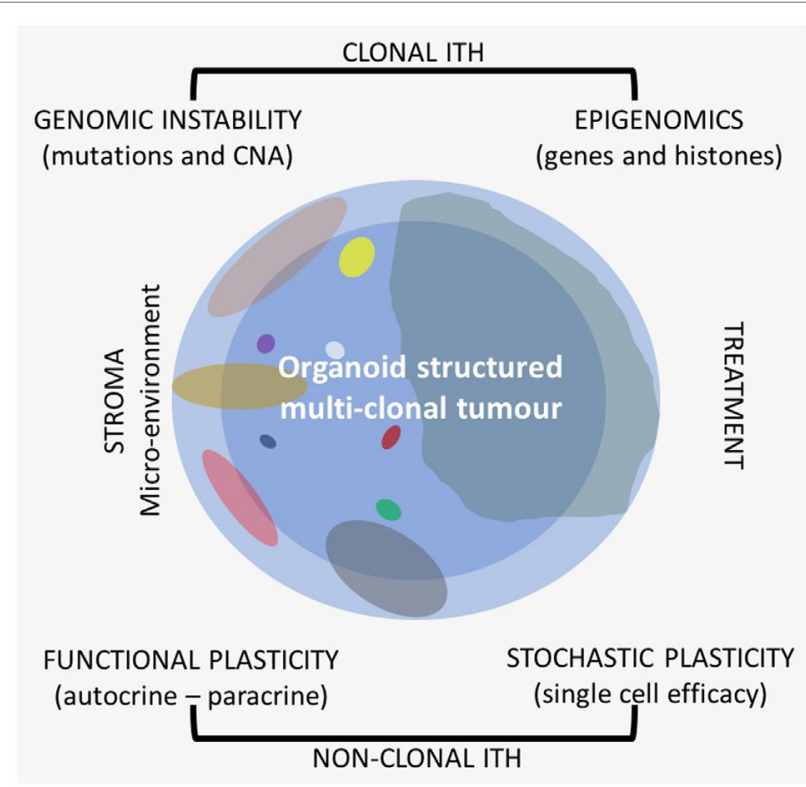

FIGURE 1 | Different types of intra-tumor heterogeneity (ITH) in an organoid structured multiclonal tumor: the primary clone is blue (the peripheral area is light blue and the central one is darker), the other clones are of different size and multicolor. interaction between clonal genomic instability of cancer and the microenvironment leads to a nonclonal phenotypical functional plasticity which is related to autocrine and paracrine interactions with a quite wide phenotype range. Besides phenotypical functional plasticity, ITH is also related to a stochastic type of plasticity that can affect any single cell. Even in cell lines, each cell is different from the others with respect to efficiency and efficacy of the single cell machinery with variable time and level of gene expression.

Methodologically, to study patients, two separate phases should be distinguished: one should include more or less localized tumors for which surgical treatment is possible; the second one should involve advanced cancer and/or cancer recurrences. In the first case, a high level of molecular information to perform an effective adjuvant therapy to avoid recurrences is needed. This should be the main strategy to reduce cancer mortality together with early diagnosis procedures. The related clinical information is obtained from the analysis of primary tumor tissues: DNA, RNA, and proteins can be analyzed using extractive as well as in situ methods. The molecular methods that are applied by maintaining the morphology, such as immunohistochemistry (IHC) and/or in situ hybridization (ISH), can be specifically useful to study heterogeneity, preserving microenvironment interactions and to evaluate new types of therapy, such as immunotherapy (15).

As for recurrences, nowadays, an important tool, the "liquid biopsy," is available. Cell-free plasma DNA (ctDNA) can be a useful instrument to analyze in those cases, giving important information for a more tailored treatment (16). New research methods and a closer clinical application are also appealing for circulating tumor cells (CTCs). This type of analysis gives further information especially on heterogeneity (17).

Intra-tumor heterogeneity analysis is the key for more efficient treatments of cancer in our patients, but only if highly reproducible clinical research is performed. We should consider that different aspects of ITH could be themselves one of the major sources of research irreproducibility, together with preclinical conditions of the biological materials and standardization of the analytical methods $(11,12)$.

\section{MORPHO-HISTOLOGICAL ITH}

Quite often, different histological patterns are present in cancer, which are related to different levels of differentiation and to metaplastic changes. It was shown in lung cancer that the different histological patterns of the same tumor can correlate with different molecular alterations (18). Even the same histotype of tumor can have several levels of differentiation, related to a different grade of atypical cells that is measured by nuclear variation and other characteristics. A good example of a standardized analysis of cancer histological heterogeneity is prostate cancer. The Gleason score enables to measure the different levels of differentiation in a tumor and can give a specific grade, which is closely related to prognosis (19).

There is a relationship between histological pattern and molecular alterations. It was shown that in lung adenocarcinomas, the mutant allele frequencies were higher in solid areas of the same tumor (18). 
The other important issue for the morphological pattern of a tumor is to consider distinct areas of the same tumor. Sampling from the border of the tumor, including the surrounding stroma and the sub-border in comparison with the central part of the tumor, can give distinctive information (Figures 1 and 2). Often, the border has a higher level of cellularity and higher neoangiogenesis than the central part, and it is well known that gene expression differs in central or periphery location (10). At present, the tissue area of the tumor submitted to molecular analysis is hardly ever disclosed in the reports, and this could be one of the major issues referred to the low level of reproducibility not only of medicobiological research but also of diagnostics. Specific and standardized types of multiple sampling in larger tumors and microdissection in multiple sites in smaller tumors are the main tools that can improve reproducibility of molecular analysis in solid tumors, also taking ITH into account.

\section{CLONAL ITH}

For many years, it has been reported that tumor progression is associated with clonal evolution of the tumor cells (20). More recently, it has been clearly shown that this is the case in cancer (21). Genetic clonal evolution of cancer is related to genomic instability as a major feature of the carcinogenetic process. In recent literature, a wide description of specific genetic mechanisms involved in DNA instability has been documented. There is evidence on increased altered DNA replication when polymerases $\mathrm{E}$ or D are mutated (Pol-E) (22), or mismatched repair genes are mutated, or their promoters are methylated [microsatellite instability (MSI)] (23), or a chromosomal instability (CIN) with CNAs is present (24-26). Those mechanisms, once described in specific tumor types, are now reported to be differently related to progression and prognosis in several tumors, such as in cancer of endometrium, colon, pancreas, breast, lung, prostate, and kidney. Pol-E and MSI are connected with high ITH associated with a high number of DNA mutations (called ultra-mutated and hypermutated tumors, respectively) and with infiltration of tumor lymphocytes (TIL) due to the presence of a multitude of neo-antigens (27). Those tumors are associated with a better prognosis, especially for Pol-E. Neo-antigens are not only related to driver mutations but also related to passenger mutations, showing that also the latest are directly involved in cancer progression. On the other hand, a high level of CNA is also related to ITH, but in this case, TILs are rarer and the prognosis tends to be worse (27-34). Recently, it has also been shown in pancreatic cancer that CNAs increase in number from primary tumor to local lymph nodes, to distant metastases (35).

Clonal cancer evolution is related not only to DNA structural alterations involving protein coding genes but also to epigenetic mechanisms that are at the basis for altered gene expression. Noncoding gene alterations can be drivers in cancer progression $(36,37)$. Hypomethylation of DNA in cancer cells can trigger cancer-germline genes with their activation (38) and the hypomethylation of intra-genomic endo-parasitic DNA repeats such as $\mathrm{L} 1$ with their reactivation and retrotransposition can increase genomic instability $(39,40)$. L1 hypomethylation is present in NSCLC already in stage I tumors (41) and is related to worse survival in colon cancer stage II (42).

It is well known that tumor-suppressor genes can be methylated at the promoter level with loss of gene expression as one of the basic mechanisms of tumor progression, and this alteration can be transmitted to the next cell generations (43, 44). Methylation patterns in lung adenocarcinomas are present with higher ITH as compared with somatic mutation in the same

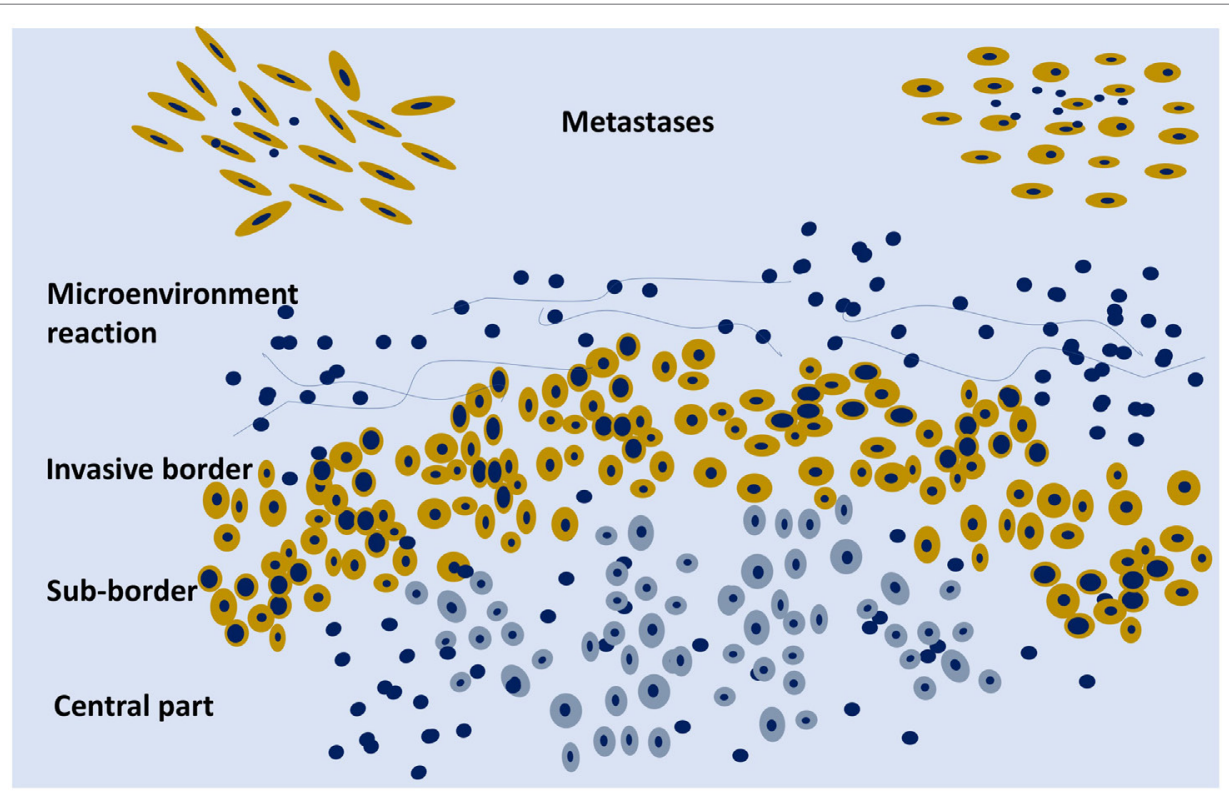

FIGURE 2 | Schematic representation of an organoid tumor with peripheral cells (yellow) surrounded by stroma components and phenotypically different from the central cells (gray). On the upper part, two metastases are schematically represented. 
tumors, suggesting to be later events in cancer progression (45). A CpG island methylator phenotype first described in colorectal cancer (46) was then reported in many different types of cancer as a generic disruption to epigenetic regulation $(47,48)$. Recently, it was shown that genome-wide methylation analysis can define pancreatic cancer subtypes (49).

Also, on the protein level, histone modification through methylation, deacetylation, phosphorylation, and ubiquitination can again silence tumor-suppressor genes or activate oncogenes (50-52). A new pathway was discovered in PTEN-deficient breast and prostate cancers with methylation of histone $\mathrm{H} 3 \mathrm{~K} 4 \mathrm{me} 3$ that activates TNFa/NF-kB (53).

All the clonal alterations can be spatial-within the primary tumor (Figures 2 and $\mathbf{3 A}$ ), sometimes involving few cells with difficulties for the specific detection-and temporal, with the acquisition of new alterations (54). They can be defined as ubiquitous or truncal when, as driver mutations, they are involved in the carcinogenetic process from the initial phases, or shared, when they are shared by several different clones, and private, when they are specific of a single clone (21).

Clonal evolution is closely related to the applied cancer treatments; ITH is connected with biological therapy resistance by inducing the development of minor resistant clones $(55,56)$. Cytotoxic therapy also influences ITH with the risk to select more aggressive clones $(57,58)$.

\section{NONCLONAL ITH}

One of the major sources of functional gene expression ITH is related to the interaction of cancer cells with the microenvironment (Figure 1). There is an autocrine interaction within the same clone cells, among different clones in a synergistic and antagonistic way (59-61), and a paracrine interaction with stroma components. All are related to clonal evolution and tumor progression (62). The microenvironment influences cell phenotype in any type of clones; the interaction can vary from area to area of the primary tumor, and the stroma of the tumor could be heterogeneous itself (63-67). All these complex interactions are known as functional phenotypic plasticity, which is well recognized by pathologists that study, e.g., epithelial-mesenchymal transition (EMT) or as stemness of cancer cells (68). Intermediate expression patterns of cells in a different functional status can be recognized with the use of specific biomarkers showing the continuous evolving

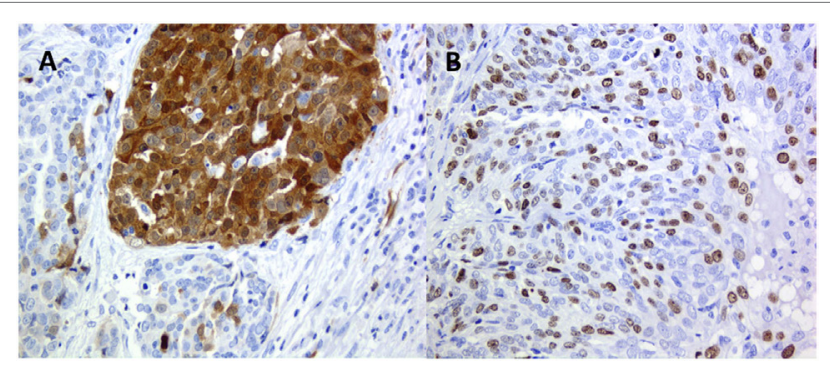

FIGURE 3 | High-grade ovary serus carcinoma: (A) p16 clonal type intra-tumor heterogeneity (ITH) and (B) Ki67 stochastic plasticity. functional plasticity in cancer (69). There is not only the paracrine influence among cancer and stroma cells but also some kind of interaction with the different collagen types of the stroma that can also have an impact on treatment outcomes (70).

Clonal genetic alterations and the microenvironment influence each other: DNA alterations are permissive for the growth of cancer cells, but the microenvironment may favor the development of specific clones in comparison with others and the microenvironment may change in time.

In thyroid cancer, the close association of EMT and cancer stem-like cells (CSCs) as well as the role of exosomes and of the microenvironment in the metastatic phenotype were shown (71).

A crucial selection is driven by the specific microenvironment induced by clinical treatments. The selection and the development of new prevalent clones are likely to be driven by clones resistant to therapy, and the new treatment associated with the microenvironment drives the clonal genetic evolution. This gives an idea of how the different clonal and nonclonal interactions are closely related in the clinical progression and in a specific way for any single patient. Recently, it has been suggested that tumors should be classified in a different way considering ITH and its development. Tumors can be characterized by the level of clonal evolution over time and its interaction with the resources available at the microenvironment level (72).

A common phenomenon recognized, e.g., in immune-histochemical analysis, is that positivity for a specific antigen varies from cell to cell in the same tumor and in the same area of the tumor, from negative cells to highly positive (Figure $\mathbf{3 B}$ ). This is related to another type of nonclonal ITH, the so-called stochastic plasticity (11-13). Each cell even in a cell line in culture has different efficacy on the transcriptional and translational level (73), and chaperone proteins such as Hsp90 may modulate phenotypic response. As a consequence, any phenotypic expression of a normal or an altered genome is further modulated differently in every single cell (74). Stochastic plasticity has been recently shown to influence resistance to chemotherapy modulating the dynamics of key players in response to treatment (75). DNA damage-inducing agents increase the expression of p53 and at the same time of inhibitor of apoptosis proteins, and resistance to chemotherapy can be caused by stochastic fluctuations in protein levels (75).

Cancer should not be considered just as an agglomerate of cells, but as a functional organoid structure: the central part of the tumor can be hypoxic and the border well oxygenated with molecular metabolic exchanges between the two parts, e.g., lactate produced by the hypoxic cells can be metabolized on the mitochondrial level by the activity of lactate dehydrogenase in the peripheral cells with a higher level of oxygenation (Figure 2). There is an intra-tumor metabolic heterogeneity with different pathway activation, which has recently been defined in kidney cancer (76). Tumor hypoxia influences not only the evolution of cancer cells but also the development of the stromal microenvironment (77).

\section{ITH IN METASTATIC SPREAD}

Intra-tumor heterogeneity usually refers to intra-primary-tumor heterogeneity, but it is also related to inter- and intra-metastatic 
heterogeneity. There are several reports in literature denying significant differences between primary tumors and metastases, but this is sometimes due to an inadequate number of analyzed samples as well as to the limited number of used biomarkers $(26,78)$. Clonal selection is the main mechanism underpinning those differences (79); therefore, it easily explains the reduced intra-metastatic heterogeneity reported (80). More aggressive and lethal clones can arise from minor subclones of the primary tumor even of relatively low grade $(81,82)$.

Monoclonal seeding of metastases has recently been dueled, suggesting the possibility of polyclonal seeding (80). Intermetastatic exchange of cancer clones that evidence the potentiality of metastatic sites to act as a primary tumor was also detected (83), and this can raise relevant considerations in favor of surgical treatment of the major metastases.

A recent work has shown that in most cases of colon cancer, the subclonal origin of the local lymph-node metastases is different from the distant metastases in other organs, denying the possibility that the latest can derive from the metastatic lymph nodes as often suggested (84).

The microenvironment differs between primary tumor and metastases influencing the phenotype of tumor cells (85). Usually, clinical biomarkers are only searched for in primary tumors, although treatments and outcomes could be better defined by their analysis in the metastatic tissues (86). Furthermore, by comparing the expression of biomarkers' profile between primary and metastasis, the molecular classification of breast cancer can vary during cancer spreading in comparison with the primitive site. Clinical relevance of a specific biomarker has also been shown to vary if the biomarker was detected in the primary tumor or in the lymph-node metastasis (87).

\section{METHODS TO STUDY ITH}

\section{Sampling}

Fixed (mostly in formalin) and paraffin-embedded (FFPE) tissues are the main resource of clinical tissue, and they are the only tissues available for any patient. The methods reported hereafter are those adapted to that type of tissues.
Most genes are not involved in clonal evolution, so it is possible to obtain reproducible quantitative analysis on the mRNA level comparing multiple samples of the same tumor taken from similar tumor areas, such as the infiltration sub-border. However, this is not the case, with an important difference in gene expression, if a specific microdissected area of the tumor is compared with the analysis of the entire tumor (12). To that purpose, accurate microdissection is required for analyses. Microdissection must avoid residual normal tissues that can modify the results of the analysis, as it is shown for several prognostic clinical signatures in breast cancer. The risk category changed along with the quantity of normal gland present in the analyzed tissue (88). An accurate sampling and analysis of primary tumors can give critical clinical information also about stroma and immune-score characteristics, as it has been recently shown in colon cancer (89). For analysis of macromolecules, microdissection is essential for comparable results and the microdissected area should be described in the report.

A preliminary type of sampling standardization has been proposed for solid tumors larger than $2 \mathrm{~cm}$ for IHC and ISH analyses, and it is going to be tested in the most common tumors for a large number of cases using the current clinical immunehistochemical biomarkers (Figure 4). This procedure has been proposed for a multicentric study involving different working groups of the European Society of Pathology. Today, there is an urgent necessity to standardize sampling procedures in light of the hugely confusing data reported in the scientific literature about frequency of ITH in any type of tumor.

\section{Extractive Methods}

Nowadays, nucleic acids extraction from clinical tissues can be a reproducible practice if it is standardized and checked with specific controls for FFPE tissues $(90,91)$. For clinical research activities, also samples fixed with Bouin's solution, which has a higher nucleic acid level of degradation, can be used with specific precautions (92). To check the final progression of cancer, also autopsy tissues can be used in many cases (93-95).

Next-generation sequencing (NGS) sensitivity is high enough to detect most clonal DNA alterations. Whole-exome sequencing

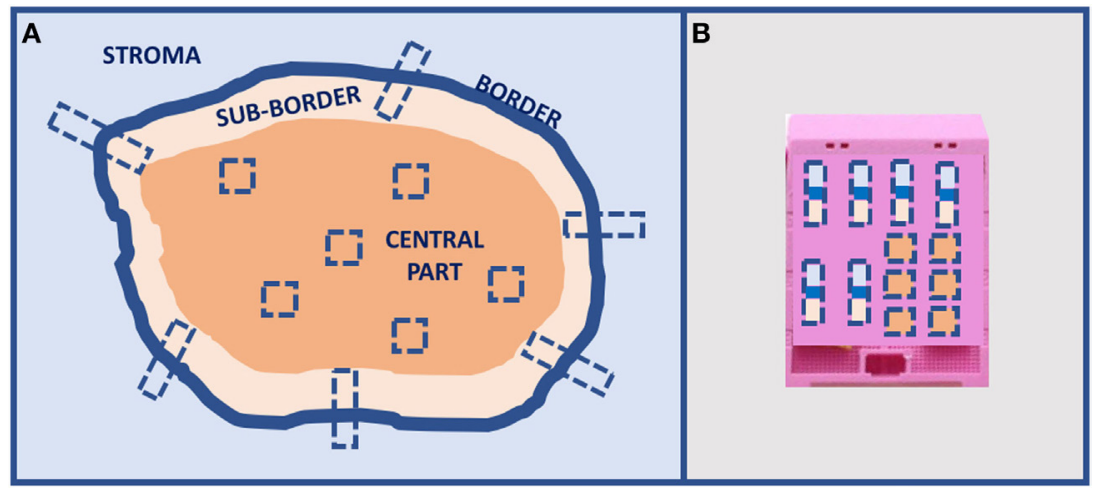

FIGURE 4 | Proposal of tumors larger than 2-cm sampling for in situ analyses: (A) multiple sampling locations and (B) organization in the inclusion block. 
(WES) of DNA in clinical tissues of primary tumors can detect several alterations present in the tumor clones that could be useful for treatments. Actionable mutations could suggest the use of specific biological drugs for that specific tumor. Actionable mutations have been detected in $90 \%$ of patients by WES (mean 4.9 per patient), a 7.5 -fold, a 2.0 -fold, and a 1.9 -fold increase over the amount obtained by using the most common NGS gene panels, namely CHPv2, OCP, and FoundationOne, respectively (96). Seventy percent of the patients have an actionable alteration with the availability of an approved drug, but in only $20 \%$, the drugs were approved for that specific type of tumor (on-label) (97). Recently, it has also been shown that not only non-synonymous mutations are involved in drug response or resistance but also synonymous ones, e.g., in response to anti-EGFR therapy in colon cancer and head and neck squamous carcinomas $(98,99)$. These figures show the importance of clinical research directly applied to patients and the necessity of new types of clinical studies.

Specific attention should be given not only to a correct technology in NGS but also to a careful interpretation and to the presence of artifacts due to fixation, such as false transition mutations (100).

DNA and RNA single cell sequencing have already been developed, and in case of DNA, it can give important information on ITH (101-103), but we should be very careful in the interpretation of RNA data because the different level of expression in every single cell could be due to stochastic plasticity.

Several methods have been used to study epigenetics in FFPE tissues. For a genome-wide methylation analysis, it is possible to use NGS-related methods or microarrays in a representative tumor sample or in different microdissected tumor areas (104), but also other specific methods, such as methylation-specific multiple ligation-dependent probe amplification, have been used (105). To quantify all the four known DNA-methylated derivatives of cytosine, namely 5 methylC, 5 hydoxymethylC, 5 formylC, and 5 carmoxylC, in FFPE tumor samples, liquid chromatography/mass spectrometry methods have been developed (104). Although it is less precise than LC/MS methods, also ELISA-based immunoquantification technology is available to detect the abovementioned methylated derivatives of cytosine in a simpler way (106).

\section{Liquid Biopsy}

The term "liquid biopsy" was originally introduced for CTCs, recovered from (liquid) peripheral blood (17). This method has rapidly improved to be shortly used also on the clinical level, the low number of collected cells being the major limit. Recently, circulating clusters of cells instead of single CTCs have been shown in breast cancer patients, which are related to higher spreading, worse prognosis, and chemo-resistance (107). This evidence could explain the presence of polyclonal seeding in the cancer metastatic process.

Liquid biopsies do not only contain CTCs but also cell-free nucleic acids and exosomes. For the presence of tumor-specific (somatic) variations in cancers, the fraction of circulating cell-free plasma tumor DNA (ctDNA), together with the larger amount of circulating cell-free DNA from normal cells, can be used as specific blood-based analyses for cancer patients' care. Thus, the use of a patient's "liquid biopsy" can allow identifying residual micro-metastatic cancer and investigating specific mutations without any invasive intervention (16). "Liquid biopsy" can theoretically offer a real-time assessment of molecular tumor genotype (qualitatively) and existing tumor burden (quantitatively) and in this way also ITH information. The major limitation for plasma tumor DNA has been the low detection rate; however, new techniques, such as digital PCR, have increased sensitivity (16). The ideal assays for liquid biopsies ctDNA would allow interrogating mutations in several genes at the same time, which represents a technical challenge because the total amount of ctDNA and its quality are both relatively low (56). The analysis of ctDNA can be helpful during the follow-up of patients to detect both truncal and private mutations. However, there is the need to better understand the contribution of each dynamic cell population in a heterogeneous tumor to ctDNA (56). It is manifest that CTCs and ctDNA can come from different and heterogeneous metastatic sites; therefore, sensitivity and reproducibility in detecting tumor range still have to be established $(108,109)$. Furthermore, in the scenario of tumor heterogeneity it should be highlighted that these low invasive methods that can be repeated many times during the follow-up, are especially useful, because the same cancer therapy can drive the selection pressure that causes clonal evolution (55).

\section{CRISPR Barcoding in Heterogeneity}

Detection of preexisting resistant subclones could be hard from a methodological point of view, because of their rarity. Sensitivity of the current methods is not enough to comprehensively consider cancer individual cells in heterogeneous cancer-cell populations (110). Recently, Guernet et al. developed a highly sophisticated CRISPR-barcoding system that enables the functional investigation of specific mutations, in a context that closely mimics the complexity of cancer (111). The high-resolution tracking of single specific cancer cells allows identifying even rare preexisting resistant subclones that can be involved in acquired resistance to therapy. Using CRISPR/ Cas9 technology, the fastest ever genome engineering technology, and specific DNA barcodes, a strategy to recapitulate and trace the emergence of subpopulations of cancer cells containing a mutation of interest has been developed. The method has already been used to study mechanisms of lung cancer cell resistance to EGFR inhibitors and to investigate on combined drug therapies. Highly complex barcodes inserted in a specific genome location have been used to simultaneously trace the fates of many thousands of genetically labeled cancer cells (111). This methodology could significantly improve the understanding of ITH and its relationship with tumor progression.

\section{Molecular Morphology}

The complexity of ITH is strictly related to the biology of the tumor, to treatment possibilities, and to recurrence probability. As a consequence, we need more sophisticated, sensitive, and morphology-related methods that can provide information on different clones or different cell types at the molecular level and their relationship with the microenvironment. Localized complex phenomena, such as tumor budding, can be studied only by 
morphology-related methods that show close localized relationship between cancer cells and stroma (65). ITH is indeed detectable not only among cancer cells but also at the tumor stroma level (112). Recently, molecular morphology has demonstrated its utility in predicting the efficacy of immunotherapy (15).

IHC for proteins and ISH for DNA and RNA can be successfully used in a reproducible way in FFPE tissues. The interplay of these methods in digital analysis is going to gain a more objective quantitative evaluation.

FISH (and other similar methods) for DNA and IHC for proteins have been performed for many years also at the clinical level, but today, we also have reproducible in situ methods for mRNAs and noncoding RNAs and developing tools to detect most genetic, genomic, and epigenetic types of alterations. ITH can be studied at the in situ level for gene amplification and CIN with FISH $(113,114)$, for single nucleotide mutation (115), for fusion transcripts detection (116), for repetitive RNAs related to hypomethylation (117), and for in situ detection of histone modification (118). For proteins, more sophisticated methods, such as matrix-assisted laser desorption/ionization imaging can be used (119). Most of those in situ techniques are not very widely diffused as methods for clinical research, and pathologists should improve their experience in this field.

Pre-analytical conditions of tissues are a basic prerequisite for reproducible results even for in situ methods. Technical CEN specifications are already available for extractive methods in FFPE tissues (DNA, RNA, and proteins) and for ctDNA from plasma as supported by SPIDIA4P EU project ${ }^{1}$ and developed by the European Committee for Standardization. ${ }^{2}$ For in situ methods, ISO documents are ongoing as described in SPIDIA4P EU project.

\section{CONCLUSION}

There is the necessity to consider ITH from a practical point of view to improve diagnosis and treatment in cancer. The major remarks could be the following:

- Clinical tumor heterogeneity on the inter- or intra-tumor level limits the utility and the application of tumor

${ }^{1}$ Standardisation and improvement of generic pre-analytical tools and procedures for in-vitro diagnostics (SPIDIA and SPIDIA4P). Available from: http://www. spidia.eu/about-the-projects/.

${ }^{2}$ European Committee for Standardization (CEN). Available from https://www. cen.eu/Pages/default.aspx.

\section{REFERENCES}

1. Uhlen M, Zhang C, Lee S, Sjostedt E, Fagerberg L, Bidkhori G, et al. A pathology atlas of the human cancer transcriptome. Science (2017) 18:357. doi: $10.1126 /$ science.aan 2507

2. Wood LD, Parsons DW, Jones S, Lin J, Sjoblom T, Leary RJ, et al. The genomic landscapes of human breast and colorectal cancers. Science (2007) 318:1108-13. doi:10.1126/science. 1145720

3. Ellsworth RE, Blackburn HL, Shriver CD, Soon-Shiong P, Ellsworth DL. Molecular heterogeneity in breast cancer: state of the science and molecular classifications based on few molecular common biomarkers.

- There is an increasing necessity to apply clinical research directly to clinics, a research performed for the today's patients with new types of studies to shorten the time for wide clinical application.

- ITH in this type of research is crucial, because it is closely related to cancer progression, therapy resistance, and recurrences.

- ITH shows different aspects in cancer, on the morphological-histological level, and on the molecular one. Molecular ITH can be divided into clonal and nonclonal. The clonal one is related to different types of genomic instability that also influence aggressiveness and treatment. The nonclonal one is functional, microenvironment related, or stochastic, notably single cell efficiency related. Clonal and microenvironment ITHs are closely connected and influence each other.

- All these types of ITH affect cancer progression and treatment efficacy and should be considered as a whole for any patient.

- At least two types of ITH methodological approaches should be considered: in surgically treated tumors, a careful analysis of tissues should drive the adjuvant therapy, and in recurrent cancer, the follow-up should consider the inclusion of blood analysis of ctDNA and, in a near future, also CTCs.

- Actionable mutations and resistance alterations should also be detected in minor clones to establish a better and tailored treatment.

- For tissues, multiple microdissection of tissues should be performed, and for larger tumors, a standardized multiple sampling procedure should be adopted to increase analysis reproducibility.

- Pathologists should improve their experience in in situ methods to better study clonal and microenvironment interactions.

\section{AUTHOR CONTRIBUTIONS}

All co-authors of this manuscript contributed for conception or design of the work (mostly GS), drafting the work or revising it critically for important intellectual content, and final approval of the version to be published.

\section{ACKNOWLEDGMENTS}

The authors wish to thank Dr. Valentina Melita for her language editing work on the manuscript.

implications for patient care. Semin Cell Dev Biol (2017) 64:65-72 doi:10.1016/j.semcdb.2016.08.025

4. Biankin AV, Piantadosi S, Hollingsworth SJ. Patient-centric trials for therapeutic development in precision oncology. Nature (2015) 526:361-70. doi:10.1038/nature15819

5. Davis C, Naci H, Gurpinar E, Poplavska E, Pinto A, Aggarwal A. Availability of evidence of benefits on overall survival and quality of life of cancer drugs approved by European Medicines Agency: retrospective cohort study of drug approvals 2009-13. BMJ (2017) 359:j4530. doi:10.1136/ bmj.j4530 
6. Schork NJ. Personalized medicine: time for one-person trials. Nature (2015) 520:609-11. doi:10.1038/520609a

7. Klement GL, Arkun K, Valik D, Roffidal T, Hashemi A, Klement C, et al. Future paradigms for precision oncology. Oncotarget (2016) 7:46813-31. doi:10.18632/oncotarget.9488

8. Woodcock J, LaVange LM. Master protocols to study multiple therapies, multiple diseases, or both. N Engl J Med (2017) 377:62-70. doi:10.1056/ NEJMra1510062

9. Garattini S, Fuso Nerini I, D'Incalci M. Not only tumor but also therapy heterogeneity. Ann Oncol (2018) 29:13-9. doi:10.1093/annonc/mdx646

10. Hlubek F, Brabletz T, Budczies J, Pfeiffer S, Jung A, Kirchner T. Heterogeneous expression of Wnt/beta-catenin target genes within colorectal cancer. Int J Cancer (2007) 121:1941-8. doi:10.1002/ijc.22916

11. Stanta G. Tissue Heterogeneity as a pre-analytical source of variability. Recent Results Cancer Res Fortschritte der Krebsforschung Progres dans les recherches sur le cancer (2015) 199:35-43. doi:10.1007/978-3-319-13957-9_4

12. Stanta G, Bonin S. A practical approach to tumor heterogeneity in clinical research and diagnostics. Pathobiology (2017). doi:10.1159/000477813

13. Stanta G, Jahn SW, Bonin S, Hoefler G. Tumour heterogeneity: principles and practical consequences. Virchows Arch (2016) 469:371-84. doi:10.1007/ s00428-016-1987-9

14. Liu F, Wang L, Perna F, Nimer SD. Beyond transcription factors: how oncogenic signalling reshapes the epigenetic landscape. Nat Rev Cancer (2016) 16:359-72. doi:10.1038/nrc.2016.41

15. McLaughlin J, Han G, Schalper KA, Carvajal-Hausdorf D, Pelekanou V, Rehman J, et al. Quantitative assessment of the heterogeneity of PD-L1 expression in non-small-cell lung cancer. JAMA Oncol (2016) 2:46-54. doi:10.1001/jamaoncol.2015.3638

16. Parsons HA, Beaver JA, Park BH. Circulating plasma tumor DNA. Adv Exp Med Biol (2016) 882:259-76. doi:10.1007/978-3-319-22909-6_11

17. Alix-Panabieres C, Pantel K. Circulating tumor cells: liquid biopsy of cancer. Clin Chem (2013) 59:110-8. doi:10.1373/clinchem.2012.194258

18. Dietz S, Harms A, Endris V, Eichhorn F, Kriegsmann M, Longuespee R, et al. Spatial distribution of EGFR and KRAS mutation frequencies correlates with histological growth patterns of lung adenocarcinomas. Int J Cancer (2017) 141:1841-8. doi:10.1002/ijc.30881

19. Epstein JI, Egevad L, Amin MB, Delahunt B, Srigley JR, Humphrey PA, et al. The 2014 International Society of Urological Pathology (ISUP) consensus conference on Gleason grading of prostatic carcinoma: definition of grading patterns and proposal for a new grading system. Am J Surg Pathol (2016) 40:244-52. doi:10.1097/PAS.0000000000000530

20. Sporn MB. The war on cancer. Lancet (1996) 347:1377-81. doi:10.1016/ S0140-6736(96)91015-6

21. Gerlinger M, Rowan AJ, Horswell S, Larkin J, Endesfelder D, Gronroos E, et al. Intratumor heterogeneity and branched evolution revealed by multiregion sequencing. N Engl J Med (2012) 366:883-92. doi:10.1056/NEJMoa1113205

22. Briggs S, Tomlinson I. Germline and somatic polymerase epsilon and delta mutations define a new class of hypermutated colorectal and endometrial cancers. J Pathol (2013) 230:148-53. doi:10.1002/path.4185

23. Yamamoto H, Imai K. Microsatellite instability: an update. Arch Toxicol (2015) 89:899-921. doi:10.1007/s00204-015-1474-0

24. Burrell RA, McGranahan N, Bartek J, Swanton C. The causes and consequences of genetic heterogeneity in cancer evolution. Nature (2013) 501:338-45. doi:10.1038/nature12625

25. Tanaka K, Hirota T. Chromosomal instability: a common feature and a therapeutic target of cancer. Biochim Biophys Acta (2016) 1866:64-75. doi:10.1016/j.bbcan.2016.06.002

26. Turajlic S, Swanton C. Metastasis as an evolutionary process. Science (2016) 352:169-75. doi:10.1126/science.aaf2784

27. van Gool IC, Eggink FA, Freeman-Mills L, Stelloo E, Marchi E, de Bruyn M, et al. POLE proofreading mutations elicit an antitumor immune response in endometrial cancer. Clin Cancer Res (2015) 21:3347-55. doi:10.1158/10780432.CCR-15-0057

28. Campbell PJ, Yachida S, Mudie LJ, Stephens PJ, Pleasance ED, Stebbings LA, et al. The patterns and dynamics of genomic instability in metastatic pancreatic cancer. Nature (2010) 467:1109-13. doi:10.1038/nature09460

29. Cancer Genome Atlas Network. Comprehensive molecular characterization of human colon and rectal cancer. Nature (2012) 487:330-7. doi:10.1038/ nature 11252
30. Cancer Genome Atlas Research Network, Kandoth C, Schultz N, Cherniack $\mathrm{AD}$, Akbani R, Liu Y, et al. Integrated genomic characterization of endometrial carcinoma. Nature (2013) 497:67-73. doi:10.1038/nature12113

31. Gerlinger M, Horswell S, Larkin J, Rowan AJ, Salm MP, Varela I, et al. Genomic architecture and evolution of clear cell renal cell carcinomas defined by multiregion sequencing. Nat Genet (2014) 46:225-33. doi:10.1038/ ng.2891

32. Hieronymus H, Schultz N, Gopalan A, Carver BS, Chang MT, Xiao Y, et al. Copy number alteration burden predicts prostate cancer relapse. Proc Natl Acad Sci U S A (2014) 111:11139-44. doi:10.1073/pnas.1411446111

33. Jamal-Hanjani M, Wilson GA, McGranahan N, Birkbak NJ, Watkins TBK, Veeriah S, et al. Tracking the evolution of non-small-cell lung cancer. $N$ Engl J Med (2017) 376:2109-21. doi:10.1056/NEJMoa1616288

34. Yates LR, Gerstung M, Knappskog S, Desmedt C, Gundem G, Van Loo P, et al. Subclonal diversification of primary breast cancer revealed by multiregion sequencing. Nat Med (2015) 21:751-9. doi:10.1038/nm.3886

35. Rausch V, Krieg A, Camps J, Behrens B, Beier M, Wangsa D, et al. Array comparative genomic hybridization of 18 pancreatic ductal adenocarcinomas and their autologous metastases. BMC Res Notes (2017) 10:560. doi:10.1186/ s13104-017-2886-0

36. Forrest ME, Khalil AM. Review: regulation of the cancer epigenome by long non-coding RNAs. Cancer Lett (2017) 407:106-12. doi:10.1016/j. canlet.2017.03.040

37. Yao X, Xing M, Ooi WF, Tan P, Teh BT. Epigenomic consequences of coding and noncoding driver mutations. Trends Cancer (2016) 2:585-605. doi:10.1016/j.trecan.2016.09.002

38. Van Tongelen A, Loriot A, De Smet C. Oncogenic roles of DNA hypomethylation through the activation of cancer-germline genes. Cancer Lett (2017) 396:130-7. doi:10.1016/j.canlet.2017.03.029

39. Madakashira BP, Sadler KC. DNA methylation, nuclear organization, and cancer. Front Genet (2017) 8:76. doi:10.3389/fgene.2017.00076

40. Scott EC, Devine SE. The role of somatic L1 retrotransposition in human Cancers. Viruses (2017) 9:131. doi:10.3390/v9060131

41. Imperatori A, Sahnane N, Rotolo N, Franzi F, Nardecchia E, Libera L, et al. LINE-1 hypomethylation is associated to specific clinico-pathological features in stage I non-small cell lung cancer. Lung Cancer (2017) 108:83-9. doi:10.1016/j.lungcan.2017.03.003

42. Swets M, Zaalberg A, Boot A, van Wezel T, Frouws MA, Bastiaannet E, et al. Tumor LINE-1 methylation level in association with survival of patients with stage II colon cancer. Int J Mol Sci (2016) 27:18. doi:10.3390/ijms180 10036

43. Heyn H, Vidal E, Ferreira HJ, Vizoso M, Sayols S, Gomez A, et al. Epigenomic analysis detects aberrant super-enhancer DNA methylation in human cancer. Genome Biol (2016) 17:11. doi:10.1186/s13059-016-0879-2

44. Olkhov-Mitsel E, Bapat B. Strategies for discovery and validation of methylated and hydroxymethylated DNA biomarkers. Cancer Med (2012) 1:237-60. doi:10.1002/cam4.22

45. Quek K, Li J, Estecio M, Zhang J, Fujimoto J, Roarty E, et al. DNA methylation intratumor heterogeneity in localized lung adenocarcinomas. Oncotarget (2017) 28(8):21994-2002. doi:10.18632/oncotarget.15777

46. Toyota M, Ahuja N, Ohe-Toyota M, Herman JG, Baylin SB, Issa JP. CpG island methylator phenotype in colorectal cancer. Proc Natl Acad Sci U S A (1999) 96:8681-6. doi:10.1073/pnas.96.15.8681

47. Hughes LA, Melotte V, de Schrijver J, de Maat M, Smit VT, Bovee JV, et al. The CpG island methylator phenotype: what's in a name? Cancer Res (2013) 73:5858-68. doi:10.1158/0008-5472.CAN-12-4306

48. Miller BF, Sanchez-Vega F, Elnitski L. The emergence of pan-cancer CIMP and its elusive interpretation. Biomolecules (2016) 6:45. doi:10.3390/ biom6040045

49. Mishra NK, Guda C. Genome-wide DNA methylation analysis reveals molecular subtypes of pancreatic cancer. Oncotarget (2017) 8:28990-9012. doi:10.18632/oncotarget.15993

50. Biswas S, Rao CM. Epigenetics in cancer: fundamentals and beyond. Pharmacol Ther (2017) 173:118-34. doi:10.1016/j.pharmthera.2017.02.011

51. Cole AJ, Clifton-Bligh R, Marsh DJ. Histone H2B monoubiquitination: roles to play in human malignancy. Endocr Relat Cancer (2015) 22:T19-33. doi:10.1530/ERC-14-0185

52. Kim KH, Roberts CW. Targeting EZH2 in cancer. Nat Med (2016) 22:128-34. doi:10.1038/nm.4036 
53. Zhao D, Lu X, Wang G, Lan Z, Liao W, Li J, et al. Synthetic essentiality of chromatin remodelling factor CHD1 in PTEN-deficient cancer. Nature (2017) 542:484-8. doi:10.1038/nature21357

54. Beksac AT, Paulucci DJ, Blum KA, Yadav SS, Sfakianos JP, Badani KK. Heterogeneity in renal cell carcinoma. Urol Oncol (2017) 35:507-15. doi:10.1016/j.urolonc.2017.05.006

55. Misale S, Yaeger R, Hobor S, Scala E, Janakiraman M, Liska D, et al. Emergence of KRAS mutations and acquired resistance to anti-EGFR therapy in colorectal cancer. Nature (2012) 486:532-6. doi:10.1038/ nature 11156

56. Vilar E, Tabernero J. Cancer: pinprick diagnostics. Nature (2012) 486:482-3. doi:10.1038/486482a

57. Donada M, Bonin S, Barbazza R, Pettirosso D, Stanta G. Management of stage II colon cancer - the use of molecular biomarkers for adjuvant therapy decision. BMC Gastroenterol (2013) 13:36. doi:10.1186/1471-230X-13-36

58. Landau DA, Carter SL, Stojanov P, McKenna A, Stevenson K, Lawrence MS, et al. Evolution and impact of subclonal mutations in chronic lymphocytic leukemia. Cell (2013) 152:714-26. doi:10.1016/j.cell.2013.01.019

59. Marusyk A, Tabassum DP, Altrock PM, Almendro V, Michor F, Polyak K. Non-cell-autonomous driving of tumour growth supports sub-clonal heterogeneity. Nature (2014) 514:54-8. doi:10.1038/nature13556

60. Tissot T, Thomas F, Roche B. Non-cell-autonomous effects yield lower clonal diversity in expanding tumors. Sci Rep (2017) 7:11157. doi:10.1038/ s41598-017-11562-w

61. Zhou H, Neelakantan D, Ford HL. Clonal cooperativity in heterogenous cancers. Semin Cell Dev Biol (2017) 64:79-89. doi:10.1016/j.semcdb.2016.08.028

62. Kleppe M, Levine RL. Tumor heterogeneity confounds and illuminates: assessing the implications. Nat Med (2014) 20:342-4. doi:10.1038/nm.3522

63. Dunne PD, Alderdice M, O'Reilly PG, Roddy AC, McCorry AMB, Richman S, et al. Cancer-cell intrinsic gene expression signatures overcome intratumoural heterogeneity bias in colorectal cancer patient classification. Nat Commun (2017) 8:15657. doi:10.1038/ncomms15657

64. Espinosa I, Catasus L, D’ Angelo E, Mozos A, Pedrola N, Bertolo C, et al. Stromal signatures in endometrioid endometrial carcinomas. Mod Pathol (2014) 27:631-9. doi:10.1038/modpathol.2013.131

65. Galvan JA, Zlobec I, Wartenberg M, Lugli A, Gloor B, Perren A, et al. Expression of E-cadherin repressors SNAIL, ZEB1 and ZEB2 by tumour and stromal cells influences tumour-budding phenotype and suggests heterogeneity of stromal cells in pancreatic cancer. Br J Cancer (2015) 112:1944-50. doi:10.1038/bjc.2015.177

66. Mani NL, Schalper KA, Hatzis C, Saglam O, Tavassoli F, Butler M, et al. Quantitative assessment of the spatial heterogeneity of tumor-infiltrating lymphocytes in breast cancer. Breast Cancer Res (2016) 29(18):78. doi:10.1186/s13058-016-0737-x

67. Shi L, Zhang Y, Feng L, Wang L, Rong W, Wu F, et al. Multi-omics study revealing the complexity and spatial heterogeneity of tumor-infiltrating lymphocytes in primary liver carcinoma. Oncotarget (2017) 8:34844-57. doi:10.18632/oncotarget.16758

68. Denisov EV, Skryabin NA, Gerashchenko TS, Tashireva LA, Wilhelm J, Buldakov MA, et al. Clinically relevant morphological structures in breast cancer represent transcriptionally distinct tumor cell populations with varied degrees of epithelial-mesenchymal transition and CD44(+)CD24(-) stemness. Oncotarget (2017) 8:61163-80. doi:10.18632/oncotarget.18022

69. Marotta LL, Almendro V, Marusyk A, Shipitsin M, Schemme J, Walker SR, et al. The JAK2/STAT3 signaling pathway is required for growth of CD44(+) CD24(-) stem cell-like breast cancer cells in human tumors. J Clin Invest (2011) 121:2723-35. doi:10.1172/JCI44745

70. Chien J, Kuang R, Landen C, Shridhar V. Platinum-sensitive recurrence in ovarian cancer: the role of tumor microenvironment. Front Oncol (2013) 3:251. doi:10.3389/fonc.2013.00251

71. Hardin H, Zhang R, Helein H, Buehler D, Guo Z, Lloyd RV. The evolving concept of cancer stem-like cells in thyroid cancer and other solid tumors. Lab Invest (2017) 97:1142-51. doi:10.1038/labinvest.2017.41

72. Maley CC, Aktipis A, Graham TA, Sottoriva A, Boddy AM, Janiszewska M, et al. Classifying the evolutionary and ecological features of neoplasms. Nat Rev Cancer (2017) 17:605-19. doi:10.1038/nrc.2017.69

73. Li GW, Xie XS. Central dogma at the single-molecule level in living cells. Nature (2011) 475:308-15. doi:10.1038/nature10315
74. Burga A, Casanueva MO, Lehner B. Predicting mutation outcome from early stochastic variation in genetic interaction partners. Nature (2011) 480:250-3. doi:10.1038/nature10665

75. Paek AL, Liu JC, Loewer A, Forrester WC, Lahav G. Cell-to-cell variation in p53 dynamics leads to fractional killing. Cell (2016) 165:631-42. doi:10.1016/j.cell.2016.03.025

76. Okegawa T, Morimoto M, Nishizawa S, Kitazawa S, Honda K, Araki H, et al. Intratumor heterogeneity in primary kidney cancer revealed by metabolic profiling of multiple spatially separated samples within tumors. EBioMedicine (2017) 19:31-8. doi:10.1016/j.ebiom.2017.04.009

77. Feron O. Pyruvate into lactate and back: from the Warburg effect to symbiotic energy fuel exchange in cancer cells. Radiother Oncol (2009) 92:329-33. doi:10.1016/j.radonc.2009.06.025

78. Brannon AR, Vakiani E, Sylvester BE, Scott SN, McDermott G, Shah RH, et al. Comparative sequencing analysis reveals high genomic concordance between matched primary and metastatic colorectal cancer lesions. Genome Biol (2014) 15:454. doi:10.1186/s13059-014-0454-7

79. Russnes HG, Navin N, Hicks J, Borresen-Dale AL. Insight into the heterogeneity of breast cancer through next-generation sequencing. J Clin Invest (2011) 121:3810-8. doi:10.1172/JCI57088

80. Wei Q, Ye Z, Zhong X, Li L, Wang C, Myers RE, et al. Multiregion whole-exome sequencing of matched primary and metastatic tumors revealed genomic heterogeneity and suggested polyclonal seeding in colorectal cancer metastasis. Ann Oncol (2017) 28:2135-41. doi:10.1093/annonc/ $\operatorname{mdx} 278$

81. Haffner MC, Mosbruger T, Esopi DM, Fedor H, Heaphy CM, Walker DA, et al. Tracking the clonal origin of lethal prostate cancer. J Clin Invest (2013) 123:4918-22. doi:10.1172/JCI70354

82. Talmadge JE, Fidler IJ. AACR centennial series: the biology of cancer metastasis: historical perspective. Cancer Res (2010) 70:5649-69. doi:10.1158/0008 5472.CAN-10-1040

83. GundemG,VanLooP,KremeyerB,AlexandrovLB,TubioJM,PapaemmanuilE, et al. The evolutionary history of lethal metastatic prostate cancer. Nature (2015) 520:353-7. doi:10.1038/nature14347

84. Naxerova K, Reiter JG, Brachtel E, Lennerz JK, van de Wetering M, Rowan A, et al. Origins of lymphatic and distant metastases in human colorectal cancer. Science (2017) 357:55-60. doi:10.1126/science.aai8515

85. Comen EA. Tracking the seed and tending the soil: evolving concepts in metastatic breast cancer. Discov Med (2012) 14:97-104.

86. Cummings MC, Simpson PT, Reid LE, Jayanthan J, Skerman J, Song S, et al. Metastatic progression of breast cancer: insights from 50 years of autopsies. J Pathol (2013) 232:23-31. doi:10.1002/path.4288

87. Bonin S, Pracella D, Barbazza R, Sulfaro S, Stanta G. In stage II/III lymph node-positive breast cancer patients less than 55 years of age, keratin 8 expression in lymph node metastases but not in the primary tumour is an indicator of better survival. Virchows Arch (2015) 466(5):571-80. doi:10.1007/ s00428-015-1748-1

88. Elloumi F, Hu Z, Li Y, Parker JS, Gulley ML, Amos KD, et al. Systematic bias in genomic classification due to contaminating non-neoplastic tissue in breast tumor samples. BMCMed Genomics (2011) 4:54. doi:10.1186/1755-8794-4-54

89. Mlecnik B, Bindea G, Kirilovsky A, Angell HK, Obenauf AC, Tosolini M, et al. The tumor microenvironment and immunoscore are critical determinants of dissemination to distant metastasis. Sci Transl Med (2016) 8:327ra326 doi:10.1126/scitranslmed.aad6352

90. Bonin S, Hlubek F, Benhattar J, Denkert C, Dietel M, Fernandez PL, et al. Multicentre validation study of nucleic acids extraction from FFPE tissues. Virchows Arch (2010) 457:309-17. doi:10.1007/s00428-010-0917-5

91. Bonin S, Stanta G. Nucleic acid extraction methods from fixed and paraffin-embedded tissues in cancer diagnostics. Expert Rev Mol Diagn (2013) 13:271-82. doi:10.1586/erm.13.14

92. Bonin S, Petrera F, Rosai J, Stanta G. DNA and RNA obtained from Bouin's fixed tissues. J Clin Pathol (2005) 58:313-6. doi:10.1136/jcp.2004.016477

93. Bonin S, Petrera F, Stanta G. PCR and RT-PCR analysis in archival postmortem tissues. In: Fuchs J, Podda M, editors. Encyclopedia of Diagnostic Genomics and Proteomics. New York: Marcel Dekker Inc. (2005). p. 985-8.

94. Carithers LJ, Ardlie K, Barcus M, Branton PA, Britton A, Buia SA, et al. A novel approach to high-quality postmortem tissue procurement: the GTEx project. Biopreserv Biobank (2015) 13:311-9. doi:10.1089/bio.2015.0032 
95. Saito T, Kondo C, Shitara K, Ito Y, Saito N, Ikehara Y, et al. Comparison of intratumoral heterogeneity of HER2 expression between primary tumor and multiple organ metastases in gastric cancer: clinicopathological study of three autopsy cases and one resected case. Pathol Int (2015) 65:309-17. doi:10.1111/pin. 12290

96. Uzilov AV, Ding W, Fink MY, Antipin Y, Brohl AS, Davis C, et al. Development and clinical application of an integrative genomic approach to personalized cancer therapy. Genome Med (2016) 8:62. doi:10.1186/s13073-016-0313-0

97. Schwaederle M, Daniels GA, Piccioni DE, Fanta PT, Schwab RB, Shimabukuro KA, et al. On the road to precision cancer medicine: analysis of genomic biomarker actionability in 439 patients. Mol Cancer Ther (2015) 14:1488-94. doi:10.1158/1535-7163.MCT-14-1061

98. Bonin S, Donada M, Bussolati G, Nardon E, Annaratone L, Pichler M, et al. A synonymous EGFR polymorphism predicting responsiveness to antiEGFR therapy in metastatic colorectal cancer patients. Tumour Biol (2016) 37:7295-303. doi:10.1007/s13277-015-4543-3

99. Tan DSW, Chong FT, Leong HS, Toh SY, Lau DP, Kwang XL, et al. Long noncoding RNA EGFR-AS1 mediates epidermal growth factor receptor addiction and modulates treatment response in squamous cell carcinoma. Nat Med (2017) 23:1167-75. doi:10.1038/nm.4401

100. Lee WC, Kopetz S, Wistuba II, Zhang J. Metastasis of cancer: when and how? Ann Oncol (2017) 28:2045-7. doi:10.1093/annonc/mdx327

101. Liu J, Adhav R, Xu X. Current progresses of single cell DNA sequencing in breast cancer research. Int J Biol Sci (2017) 13:949-60. doi:10.7150/ijbs. 19627

102. Martelotto LG, Baslan T, Kendall J, Geyer FC, Burke KA, Spraggon L, et al. Whole-genome single-cell copy number profiling from formalin-fixed paraffin-embedded samples. Nat Med (2017) 23:376-85. doi:10.1038/ nm. 4279

103. Wang J, Song Y. Single cell sequencing: a distinct new field. Clin Transl Med (2017) 6:10. doi:10.1186/s40169-017-0139-4

104. Tang J, Fang F, Miller DF, Pilrose JM, Matei D, Huang TH, et al. Global DNA methylation profiling technologies and the ovarian cancer methylome. Methods Mol Biol (2015) 1238:653-75. doi:10.1007/978-1-4939-1804$1 \_34$

105. Berbegall AP, Villamon E, Navarro S, Noguera R. Multiplex ligation-dependent probe amplification (MLPA). In: Stanta G, editor. Guidelines for Molecular Analysis in Archive Tissues. Berlin, Heidelberg: Springer (2011). p. 215-24.

106. Chowdhury B, Cho IH, Hahn N, Irudayaraj J. Quantification of 5-methylcytosine, 5-hydroxymethylcytosine and 5-carboxylcytosine from the blood of cancer patients by an enzyme-based immunoassay. Anal Chim Acta (2014) 852:212-7. doi:10.1016/j.aca.2014.09.020

107. Cheung KJ, Padmanaban V, Silvestri V, Schipper K, Cohen JD, Fairchild AN, et al. Polyclonal breast cancer metastases arise from collective dissemination of keratin 14-expressing tumor cell clusters. Proc Natl Acad Sci U S A (2016) 113:E854-63. doi:10.1073/pnas.1508541113

108. Diaz LA Jr, Bardelli A. Liquid biopsies: genotyping circulating tumor DNA J Clin Oncol (2014) 32:579-86. doi:10.1200/JCO.2012.45.2011

109. Pantel K, Diaz LA Jr, Polyak K. Tracking tumor resistance using 'liquid biopsies'. Nat Med (2013) 19:676-7. doi:10.1038/nm.3233
110. Marino FZ, Accardo M, Franco R. CRISPR-barcoding in non small cell lung cancer: from intratumor genetic heterogeneity modeling to cancer therapy application. J Thorac Dis (2017) 9:1759-62. doi:10.21037/jtd.2017. 06.27

111. Guernet A, Mungamuri SK, Cartier D, Sachidanandam R, Jayaprakash A, Adriouch S, et al. CRISPR-barcoding for intratumor genetic heterogeneity modeling and functional analysis of oncogenic driver mutations. Mol Cell (2016) 63:526-38. doi:10.1016/j.molcel.2016.06.017

112. Liu L, Mayes PA, Eastman S, Shi H, Yadavilli S, Zhang T, et al. The BRAF and MEK inhibitors dabrafenib and trametinib: effects on immune function and in combination with immunomodulatory antibodies targeting PD-1, PD-L1, and CTLA-4. Clin Cancer Res (2015) 21(7):1639-51. doi:10.1158/1078-0432. CCR-14-2339

113. Penner-GoekeS,LichtensztejnZ,NeufeldM,AliJL,AltmanAD,NachtigalMW, et al. The temporal dynamics of chromosome instability in ovarian cancer cell lines and primary patient samples. PLoS Genet (2017) 13:e1006707. doi:10.1371/journal.pgen.1006707

114. Stahl P, Seeschaaf C, Lebok P, Kutup A, Bockhorn M, Izbicki JR, et al. Heterogeneity of amplification of HER2, EGFR, CCND1 and MYC in gastric cancer. BMC Gastroenterol (2015) 15:7. doi:10.1186/s12876-0150231-4

115. Grundberg I, Kiflemariam S, Mignardi M, Imgenberg-Kreuz J, Edlund K, Micke P, et al. In situ mutation detection and visualization of intratumor heterogeneity for cancer research and diagnostics. Oncotarget (2013) 4:2407-18 doi:10.18632/oncotarget.1527

116. Kiflemariam S, Mignardi M, Ali MA, Bergh A, Nilsson M, Sjoblom T. In situ sequencing identifies TMPRSS2-ERG fusion transcripts, somatic point mutations and gene expression levels in prostate cancers. J Pathol (2014) 234:253-61. doi:10.1002/path.4392

117. Desai N, Sajed D, Arora KS, Solovyov A, Rajurkar M, Bledsoe JR, et al. Diverse repetitive element RNA expression defines epigenetic and immunologic features of colon cancer. JCI Insight (2017) 2:e91078. doi:10.1172/ jci.insight. 91078

118. Gomez D, Shankman LS, Nguyen AT, Owens GK. Detection of histone modifications at specific gene loci in single cells in histological sections. Nat Methods (2013) 10:171-7. doi:10.1038/nmeth.2332

119. Alberts D, Pottier C, Smargiasso N, Baiwir D, Mazzucchelli G, Delvenne P, et al. MALDI imaging-guided microproteomic analyses of heterogeneous breast tumors - a pilot study. Proteomics Clin Appl (2018) 12:1700062. doi:10.1002/prca.201700062

Conflict of Interest Statement: The authors declare that the research was conducted in the absence of any commercial or financial relationships that could be construed as a potential conflict of interest.

Copyright (C) 2018 Stanta and Bonin. This is an open-access article distributed under the terms of the Creative Commons Attribution License (CC BY). The use, distribution or reproduction in other forums is permitted, provided the original author $(s)$ and the copyright owner are credited and that the original publication in this journal is cited, in accordance with accepted academic practice. No use, distribution or reproduction is permitted which does not comply with these terms. 